\title{
Photoluminescence spectroscopy of trions in quantum dots: A theoretical description
}

\author{
Juan I. Climente, ${ }^{1,2, *}$ Andrea Bertoni, ${ }^{1}$ and Guido Goldoni ${ }^{1,3}$ \\ ${ }^{1}$ CNR-INFM National Center on nanoStructures and bioSystems at Surfaces (S3), Via Campi 213/A, 41100 Modena, Italy \\ ${ }^{2}$ Departament de Química Física i Analítica, Universitat Jaume I, Avda. Sos Baynat s/n, 12080 Castello, Spain \\ ${ }^{3}$ Dipartimento di Fisica, Università di Modena e Reggio Emilia, Via Campi 213/A, 41100 Modena, Italy \\ (Received 21 August 2008; revised manuscript received 27 September 2008; published 21 October 2008)
}

\begin{abstract}
We present a full configuration-interaction study of the spontaneous recombination of neutral and singly charged excitons (trions) in semiconductor quantum dots from weak- to strong-coupling regimes. We find that the enhancement of the recombination rate of neutral excitons with increasing dot size is suppressed for negative trions and even reversed for positive trions. Our findings agree with recent comprehensive photoluminescence experiments in self-assembled quantum dots [P. Dalgarno et al., Phys. Rev. B 77, 245311 (2008)] and confirm the major role played by correlations in the valence band. The effect of the temperature on the photoluminescence spectrum and that of the ratio between the electron and hole wave-function length scales are also described.
\end{abstract}

DOI: 10.1103/PhysRevB.78.155316

PACS number(s): 73.21.La, 71.35.Pq, 78.67.Hc

\section{INTRODUCTION}

Optoelectronics is one of the fields where the atomiclike properties of quantum dots (QDs) is liable of producing a breakthrough in device performance. ${ }^{1}$ Quantum-optical applications, such as single-photon sources ${ }^{2}$ and quantum logic gates, ${ }^{3-8}$ have been proposed and are currently under intense research. Progress in this research field has been made possible by a number of studies over the last years which provide understanding of exciton and multiexciton emission processes taking place in QDs. ${ }^{1,9}$ Many of these studies highlight the critical effect of Coulomb interactions among the particles confined in the QD to determine the photoluminescence (PL) response. Indeed, it has been shown that the relative spectral positions of exciton $\left(X^{0}\right)$ and charged exciton $\left(X^{ \pm n}\right)$ states can be generally inferred from the few-particle correlations. ${ }^{10-23}$

The dependence of the recombination rates of neutral exciton, biexciton, and multiexciton complexes on Coulomb interactions has been also described in a number of papers. ${ }^{24-30}$ Much less is known about the recombination rates of positively $\left(X^{+}\right)$and negatively $\left(X^{-}\right)$charged excitons (trions). ${ }^{20,31,32}$ This is nonetheless an interesting problem. From a fundamental point of view, comparing the recombination of $X^{0}, X^{+}$, and $X^{-}$provides direct insight into the different role played by electrons and holes as exciton components. From a practical point of view, trions are often the dominant species in QDs populated by exciting valence electrons into the conduction-band continuum. ${ }^{22}$ Moreover, they are involved in relevant applications of QDs such as the optical preparation of pure spin electrons ${ }^{33}$ or holes. ${ }^{34,35}$ They are also the natural source of single photons with pure circular polarization since neutral excitons are subject to electronhole exchange interaction and hence produce linearly polarized photons. Understanding the conditions which optimize the emission of trions is of primary importance for the development of such applications.

Very recently, Dalgarno et al. collected comprehensive spectroscopical data comparing the recombination rates and energies of neutral excitons and trions in self-assembled In-
GaAs QDs. ${ }^{31}$ Clear differences were observed upon charging $X^{0}$ with an additional electron or hole, which were qualitatively consistent with a picture of where electrons are in the strong confinement regime while holes are not. Owing to their larger mass, holes seemed to be in an intermediate confinement regime where the influence of Coulomb correlations may be significant. ${ }^{17}$

In this work, we provide theoretical assessment on this subject. We perform a full configuration-interaction (FCI) study to calculate the recombination rates of $X^{0}, X^{+}$, and $X^{-}$ in QDs with different confinement strength. With increasing QD size, $X^{0}, X^{+}$, and $X^{-}$display different trends that can be traced back to the interplay among the different Coulomb interaction terms in the system (electron-electron, hole-hole, electron-hole). In particular, we obtain an enhanced PL for $X^{0}$ with increasing dot size-a result well known from previous studies ${ }^{25-27}$ - but then show that electron-electron repulsion partially quenches the emission efficiency for $X^{-}$. For $X^{+}$the quenching is even larger due to the strong hole-hole correlation, and the reverse trend (decreasing PL with increasing dot size) is obtained. Indeed, holes are found to be significantly correlated even in typical self-assembled structures, which lead to spectral features in agreement with Ref. 31 . We also investigate the effect of the different lateral extension of single-particle electron and hole wave functions on the PL spectrum of trions, and show that this electronhole asymmetry may bring about qualitative changes in both the relative spectral positions and the relative recombination rates of $X^{0}, X^{+}$, and $X^{-}$. Finally, we predict that hole-hole correlations may be reflected in a peculiar temperature dependence of the $X^{+}$line, which does not show up for neutral and negatively charged excitons.

\section{THEORY}

We use a Hamiltonian describing $N_{e}$ electrons and $N_{h}$ heavy holes confined in a $\mathrm{QD}$,

$$
\mathcal{H}=\mathcal{H}_{e}^{N_{e}}+\mathcal{H}_{h}^{N_{h}}+V_{e h}^{N_{e} N_{h}} .
$$

Here $\mathcal{H}_{e}^{N_{e}}$ and $\mathcal{H}_{h}^{N_{h}}$ are the electron and hole effective-mass Hamiltonians, respectively, including intraband interactions, 


$$
\begin{aligned}
\mathcal{H}_{e}^{N_{e}} & =\sum_{i=1}^{N_{e}}\left[\frac{\mathbf{p}_{i}^{2}}{2 m_{e}^{*}}+V_{e}\left(\rho_{i}, z_{i}\right)+\sum_{j<i} \frac{e^{2}}{\epsilon\left|\mathbf{r}_{e}^{i}-\mathbf{r}_{e}^{j}\right|}\right], \\
\mathcal{H}_{h}^{N_{h}} & =\sum_{i=1}^{N_{h}}\left[\frac{\mathbf{p}_{i}^{2}}{2 m_{h}^{*}}+V_{h}\left(\rho_{i}, z_{i}\right)+\sum_{j<i} \frac{e^{2}}{\boldsymbol{\epsilon}\left|\mathbf{r}_{h}^{i}-\mathbf{r}_{h}^{j}\right|}\right],
\end{aligned}
$$

while $V_{e h}^{N_{e} N_{h}}$ is the Coulomb interaction term between electrons and holes,

$$
V_{e h}^{N_{e} N_{h}}=-\sum_{i=1}^{N_{e}} \sum_{j=1}^{N_{h}} \frac{e^{2}}{\epsilon\left|\mathbf{r}_{e}^{i}-\mathbf{r}_{h}^{j}\right|} .
$$

In the above expression, $m_{\alpha}^{*}$ and $V_{\alpha}(\rho, z)$ are the effective mass and the single-particle QD confinement potential, respectively, which is in general different for the two types of carriers. $\epsilon$ is the dielectric constant of the QD medium and $e$ is the electron charge. We neglect the electron-hole exchange interaction. This does not affect the singlet states of the trions we will deal with and adds only a fine structure to the exciton spectrum which is not relevant for our study. ${ }^{14,36}$

We consider a separable confining potential with cylindrical symmetry, $V(\rho, z)=V(\rho)+V(z)$. In the in-plane direction we take a parabolic confinement, $V(\rho)=1 / 2\left(m^{*} \omega^{2} \rho^{2}\right)$, with $\omega$ as the characteristic frequency. This yields Fock-Darwin (FD) single-particle states for electrons and holes, ${ }^{9}$ which provide a transparent yet fairly accurate starting point in describing the optics of QDs with different confinement regimes, from weakly confined (etched) (Ref. 37) to strongly confined (self-assembled). ${ }^{38,39}$ In the vertical direction, the potential $V(z)$ is defined by a rectangular quantum well provided by the band offset between the QD and barrier materials. The quantum well eigenstates are derived numerically.

The $N_{e}$-electron and $N_{h}$-hole states are calculated independently using a two-step FCI approach in the basis of the FD single-particle states. First, we diagonalize $\mathcal{H}_{e}^{N_{e}}$ and $\mathcal{H}_{h}^{N_{h}}$ exactly, following the FCI method described in Ref. 40. The resulting few-electron $\left(\Psi^{N_{e}}\right)$ and few-hole $\left(\Psi^{N_{h}}\right)$ states contain an exact description of the intraband correlations. Second, Hartree products of the correlated electron and hole states obtained in the first step are used to represent the electron-hole term $V_{e h}^{N_{e} N_{h}}$, which is diagonalized exactly to describe interband correlations. This method provides fully correlated excitonic states $\left(X^{N_{e} N_{h}}\right)$ which are needed for an accurate estimate of the recombination probability ${ }^{12,27,28}$ and energy. ${ }^{10-19}$ Moreover, it gives direct estimates of $V_{e h}^{N_{e} N_{h}}$, which allows us to study the renormalization of the electronhole attraction upon charging with additional electrons or holes.

The PL spectra are calculated within the dipole approximation and Fermi's golden rule. ${ }^{9}$ The recombination probability from an initial state $\left|X^{N_{e} N_{h}}, i\right\rangle$ to a final state $\left|X^{N_{e}-1 N_{h}-1}, f\right\rangle$ with one less electron-hole pair, at an emission frequency $\omega$, is then given by:

$$
\begin{aligned}
\mathcal{P}_{f \leftarrow i}(\omega)= & \left|\left\langle X^{N_{e}-1 N_{h}-1}, f|\hat{\mathcal{P}}| X^{N_{e} N_{h}}, i\right\rangle\right|^{2} f_{i}(T) \delta\left(E_{X^{N} e^{N_{h}}}^{i}\right. \\
& \left.-E_{X^{N_{e}-1 N_{h}-1}}^{f}-\hbar \omega\right) .
\end{aligned}
$$

Here $\hat{\mathcal{P}}$ is the polarization operator, $\hat{\mathcal{P}}=\sum_{r s} e_{r_{\sigma}} h_{s_{-\sigma}}\langle r \mid s\rangle$, where $e_{r_{\sigma}}$ is the annihilation operator for an electron with spin $\sigma$ in the FD state $r, h_{s_{-\sigma}}$ is the annihilation operator for a hole with the opposite spin in the FD state $s$, and $\langle r \mid s\rangle$ is the overlap between the two states. The $\delta$ function describing the energy resonance condition is replaced in practice by a Lorentzian curve with bandwidth $b=0.5 \mathrm{meV}$. We assume thermal equilibrium for the initial states so that $f_{i}(T)$ is the $i$-state Fermi distribution function at temperature $T$. Unless otherwise stated, only the lowest-energy (fundamental) transition is studied as it is the strongest. This transition involves the ground states of $X^{N_{e} N_{h}}$ and $X^{N_{e}-1 N_{h}-1}$. For low temperature, this means that the recombination involves essentially $s$ shells of the FD spectrum. Thus, the differences in the behavior of the excitonic complexes we study do not arise from different symmetries of the occupied orbitals but simply from the different correlations in each case.

To compare with the notation in related experiments, ${ }^{31}$ the energies of the transitions can be written as

$$
\begin{gathered}
E_{\mathrm{PL}}\left(X^{0}\right)=E_{X^{0}}=E_{e}^{s}+E_{h}^{s}+V_{e h}^{11}, \\
E_{\mathrm{PL}}\left(X^{+}\right)=E_{X^{+}}-E_{h}^{s}=E_{e}^{s}+E_{h}^{s}+V_{h h}+2 V_{e h}^{12}, \\
E_{\mathrm{PL}}\left(X^{-}\right)=E_{X^{-}}-E_{e}^{s}=E_{e}^{s}+E_{h}^{s}+V_{e e}+2 V_{e h}^{21} .
\end{gathered}
$$

Here $E_{X^{0}}, E_{X^{+}}$, and $E_{X^{-}}$are the ground-state energies of the neutral, positively charged, and negatively charged excitons, respectively. $E_{e}^{s}\left(E_{h}^{s}\right)$ is the energy of the single-particle $s$ orbitals for electrons (holes). $V_{e e}\left(V_{h h}\right)$ is the electronelectron (hole-hole) repulsion energy, which we calculate as the difference in energy between the two-electron (two-hole) ground state with and without Coulomb interaction. Likewise, $V_{e h}^{N_{e} N_{h}}$ is the electron-hole attraction energy, which we calculate as the difference in energy between the excitonic species with and without including Eq. (3) term in the Hamiltonian. Note that $V_{e h}^{N_{e} N_{h}}$ depends on the number of carriers of each type in the QD.

\section{RESULTS}

We consider an InGaAs/GaAs QD of height $2.5 \mathrm{~nm}$. In this material, the electron (hole) effective mass is $m_{e}^{*}=0.05$ $\left(m_{h}^{*}=0.45\right)$, the conduction (valence) band is $350(200) \mathrm{meV}$, and the dielectric constant is $\epsilon=12.9$. While this QD structure compares well to Ref. 31 samples, we note that similar findings are obtained if GaAs/AlGaAs QDs are studied. The $N_{e}$ electron and $N_{h}$ hole correlated states are calculated separately using a FCI built on all the possible Slater determinants which can be formed from the 56 lowest-energy FD spin orbitals. The excitonic states are then calculated with a basis that includes the Hartree products formed by several tens of low-energy few-electron and few-hole states. 


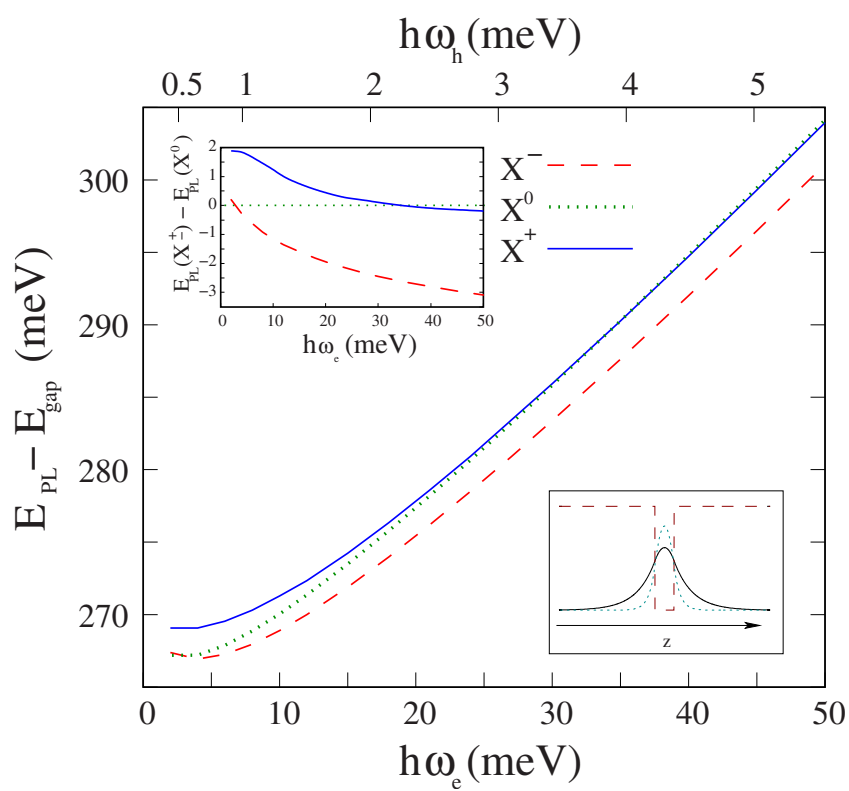

FIG. 1. (Color online) Recombination energies versus lateral confinement. The electron (hole) confinement frequency is given in the bottom (top) axis. The characteristic length is set to be the same for both kinds of particles. Upper-left inset: Energy shifts with respect to the recombination energy of $X^{0}$. Lower-right inset: Electron (solid line) and hole (dotted line) single-particle wave functions in the quantum well (dashed line).

\section{A. Electron and hole wave functions with the same lateral extension}

In a first set of calculations, we vary the confining frequency of the electron and that of the hole, ensuring that the two kinds of particles have the same confinement length in the plane, $\sqrt{\hbar / m_{e}^{*} \omega_{e}}=\sqrt{\hbar / m_{h}^{*} \omega_{h}}$. This is known to be often the case in self-assembled QDs. ${ }^{11,14}$ Note that in this way, the single-particle electron and hole wave functions are symmetric in the in-plane direction-the relevant one for the Coulomb-induced configuration mixing-and all the differences in the correlation regime can be traced back to the different level spacing, which is obviously denser for holes. Electron and hole wave functions are still different along the vertical direction, the hole being more confined due to its larger mass, as shown in the lower inset of Fig. 1. This electron-hole asymmetry, which is present in most kinds of epitaxial QDs, ${ }^{14,37}$ reduces the electron-hole overlap and hence tends to make the electron-hole attraction $\left(V_{e h}^{11}\right)$ smaller than electron-electron $\left(V_{e e}\right)$ and hole-hole $\left(V_{h h}\right)$ repulsions, which avoids artificial cancellations ${ }^{10}$ of the binding energies of trions obtained when $\left|V_{e h}^{11}\right|=\left|V_{e e}\right|=\left|V_{h h}\right|$.

In Fig. 1 we compare the recombination energies of the excitonic complexes for variable lateral size of the QD. For most confinement regimes, the relative energies agree with reported measurements in self-organized QDs of different sizes $^{23,31}$ and early theoretical studies: ${ }^{10,41} X^{+}$is blueshifted with respect to $X^{0}$ while $X^{-}$is redshifted. However, we also find the possibility of deviations from the usual sequence in the strong and weak confinement regimes. This is better seen in the upper inset of the figure, which represents the energy shifts of $X^{+}$and $X^{-}$with respect to $X^{0}: X^{+}$becomes redshifted

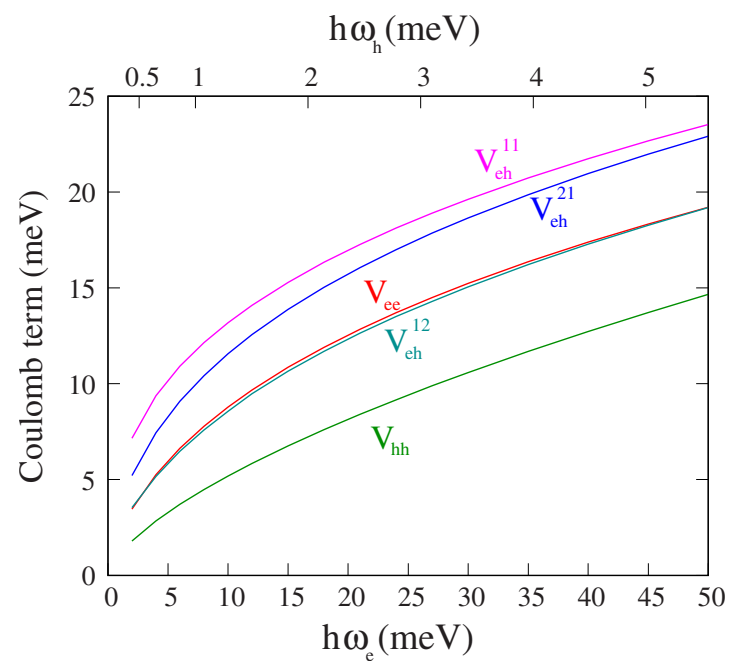

FIG. 2. (Color online) Absolute value of the Coulomb terms contributing to the exciton and trion energies of Fig. 1 [see Eq. (5)].

for very strong confinement $\left(\hbar \omega_{e}>40 \mathrm{meV}\right)$ (Ref. 41) while $X^{-}$becomes blueshifted for very weak confinement.

The result above can be rationalized by comparing the different Coulomb terms contributing to the recombination energies [see Eq. (5)], which are shown in Fig. 2. The redshift of $X^{-}$occurs because $E_{\mathrm{PL}}\left(X^{-}\right)-E_{\mathrm{PL}}\left(X^{0}\right)=V_{e e}-2 V_{e h}^{21}$ $+V_{e h}^{11}<0$, and the blueshift of $X^{+}$because $E_{\mathrm{PL}}\left(X^{+}\right)-E_{\mathrm{PL}}\left(X^{0}\right)$ $=V_{h h}-2 V_{e h}^{12}+V_{e h}^{11}>0$. It is worth noting a few points regarding the Coulomb terms in the figure: (i) $V_{e e}>V_{h h}$ for all confinement strengths. This is in spite of the heavy mass of holes, which should lead to $V_{e e}<V_{h h}$ in a perturbational approach. $^{31,42}$ This is because, unlike electrons (which have too much kinetic energy), holes are able to localize well apart in the dot, thus minimizing Coulomb repulsion (see Fig. 4). In other words, holes are strongly correlated, even in strongly confined QDs $\left(\hbar \omega_{e}=50 \mathrm{meV}\right)$. (ii) $V_{e h}^{11}$ decreases upon the inclusion of an additional carrier $\left(V_{e h}^{11}>V_{e h}^{21}>V_{e h}^{12}\right)$. This is because the Coulomb repulsion separates the two identical carriers, thus reducing the overlap with the other kind of carrier (which remains in the center of the dot). At $\hbar \omega_{e}=30 \mathrm{meV}$, the addition of one electron to form $X^{-}$reduces the Coulomb attraction in about $5 \%$, close to experimental estimates. ${ }^{31}$ The addition of one hole, according to our prediction, has a much stronger effect as the Coulomb attraction is reduced in about $20 \%$. This is another signature of the strong hole correlation. (iii) In general, $V_{e h}^{N_{e} N_{h}}$ is larger (in modulus) than $V_{e e}$ and $V_{h h}$. This is surprising if we recall that the asymmetry of the electron and hole wave functions (in the vertical direction) reduces the electron-hole overlap. This is again due to electronic correlations, which enable important redistributions of the charge in the QD through configuration mixing, maximizing attractions $\left(V_{e h}^{N_{e} N_{h}}\right)$ and minimizing repulsions $\left(V_{e e}, V_{h h}\right)$. A paradigm is the fact that $\left|V_{e h}^{11}\right|$ is clearly larger than $\left|V_{e e}\right|$.

The above observations are in qualitative agreement with the data inferred in Ref. 31 (and extend the predictions toward other confinement regimes). The only discrepancy is in point (i): while we predict $V_{e e}>V_{h h}$ they estimate $V_{e e} \sim V_{h h}$. This may be due to the uncertainty in the confinement potential (for example, a smoother potential in the $z$ direction 


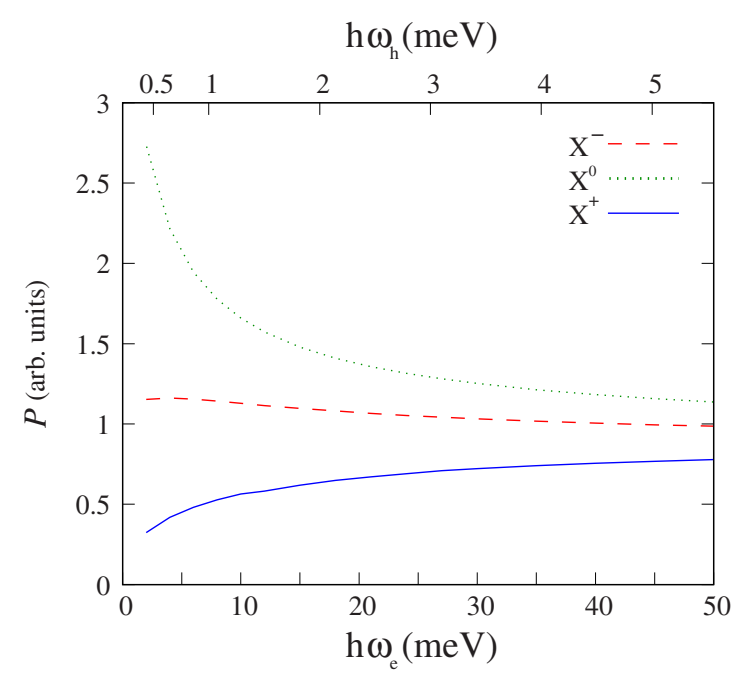

FIG. 3. (Color online) Recombination probability versus lateral confinement for the exciton and trion states of Fig. 1.

would reduce the electron-hole asymmetry) or in the experimental parameters. Note however that the values of the Coulomb terms in our calculations are obtained from the recombination energies of Fig. 1-which match well the experiment—without further approximations or assumptions.

We next investigate the recombination probability of $X^{0}$, $X^{+}$, and $X^{-}$. Figure 3 represents the recombination probability for different lateral confinement frequency and temperature $T=0 \mathrm{~K}$. The recombination probabilities always follow the relation $\mathcal{P}\left(X^{0}\right)>\mathcal{P}\left(X^{-}\right)>\mathcal{P}\left(X^{+}\right)$, in agreement with the experiment. ${ }^{31,43}$ In particular, in the strong confinement regime the recombination probability functions are rather flat and obey $\mathcal{P}\left(X^{0}\right) / \mathcal{P}\left(X^{+}\right) \approx 1.2$ and $\mathcal{P}\left(X^{0}\right) / \mathcal{P}\left(X^{-}\right) \approx 1.7$, close to the average experimental values of 1.25 and 1.58 . As the confinement strength decreases, $\mathcal{P}\left(X^{0}\right)$ increases very rapidly. This behavior is in agreement with previous predictions $\mathrm{s}^{25-27}$ and is due to the increasing electron-hole correlations, an effect which is sometimes referred to as exciton superradiance. ${ }^{44} \mathrm{~A}$ different behavior is however observed for trions. $\mathcal{P}\left(X^{-}\right)$increases only slowly. This is an indication that the repulsive electron-electron correlation partially compensates for the electron-hole attraction. On the other hand, $\mathcal{P}\left(X^{+}\right)$decreases instead of increasing. This is because hole-hole correlations dominate over electron-hole ones, and this reduces the overall electron-hole overlap.

The strong effect of hole correlations upon the chargedensity shape can be visualized in Fig. 4. The left panel shows the normalized charge densities of the particles which constitute the $X^{+}$complex while the right panel shows that of the $X^{-}$one. The relevant result is that, for $X^{+}$, the hole with spin down has a dip in the center, where the electron lies. This is the signature of hole correlations. Clearly, no such signature is observed for the pair of electrons in $X^{-}$. Since the spin-down hole is the one that recombines with the spin-up electron, the low PL of $X^{+}$reported in Fig. 3 is readily understood. This also explains the small value of $V_{h h}$ as compared to $V_{e e}$, discussed in Fig. 2.

We have tested that the effects of hole correlation discussed in this section hold also when only the lowest 20 FD spin orbitals are included in the FCI. Therefore, we do not
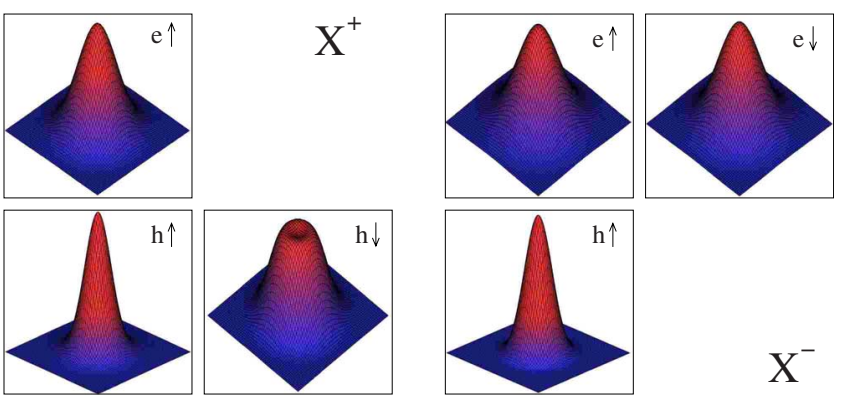

FIG. 4. (Color online) Charge density of the particles constituting the trion complexes: $X^{+}$(left panel) and $X^{-}$(right panel). The pictures correspond to a QD with $\hbar \omega_{e}=30 \mathrm{meV}$. e and h stand for electron and hole, and the arrows indicate the spin. The charge densities are averaged over $z$. For $X^{+}$one of the holes exhibits a dip in the center-the position of the electron-due to the hole-hole repulsion and the strong hole correlation. This does not happen for electrons in $X^{-}$.

expect the results to change qualitatively in QDs where the confining potential deviates from parabolicity at high energies.

\section{B. Electron and hole wave functions with different lateral extension}

So far we have assumed that the single-particle electron and hole wave functions have about the same length in the plane. As mentioned above, this is a good description for usual self-assembled InGaAs QDs (Ref. 14) but not necessarily for other kinds of QDs. This is because electrons and holes feel the band-offset potentials, the strain, and the composition fluctuations in a different way. As a result, hole wave functions in the in-plane direction may be more or less delocalized than electron ones (the tighter localization in the vertical direction is more robust because the strong confinement overrides all other effects). ${ }^{14,45}$ In quantum wires this asymmetry has been shown to strongly influence the properties of trions. ${ }^{46}$ In this section, we investigate its effect on our previous results.

We consider a QD with fixed electron confinement $\hbar \omega_{e}$ $=30 \mathrm{meV}$, which is close to the average value in Ref. 31 samples, and then vary the hole confinement frequency. The recombination energies of $X^{0}, X^{-}$, and $X^{+}$in this system are illustrated in Fig. 5. For $\hbar \omega_{h}=3.33 \mathrm{meV}$ (dashed vertical line), the electron and hole wave functions have the same extension. This is the case studied above, and the result is that of the experiments: $X^{+}$is slightly blueshifted with respect to $X^{0}$ while $X^{-}$is visibly redshifted. If we move to the right $\left(\hbar \omega_{h}>3.33 \mathrm{meV}\right)$, the hole wave function is more localized than that of the electron. While this barely affects the redshift of $X^{-}$, for $\hbar \omega_{h} \approx 4-5.5 \mathrm{meV}$ the blueshift of $X^{+}$is suppressed. About $25 \%$ of the QDs investigated in Ref. 31 exhibited this deviation, which suggests that in such dots holes were slightly more localized than electrons. Conversely, if we move to the left $\left(\hbar \omega_{h}<3.33 \mathrm{meV}\right)$, the hole wave function is less localized than that of the electron [as in pure InAs QDs (Ref. 14)]. Note that this is a regime of very strong hole correlation. It may then occur that $X^{-}$is redshifted (this was never observed in Ref. 31 samples). 


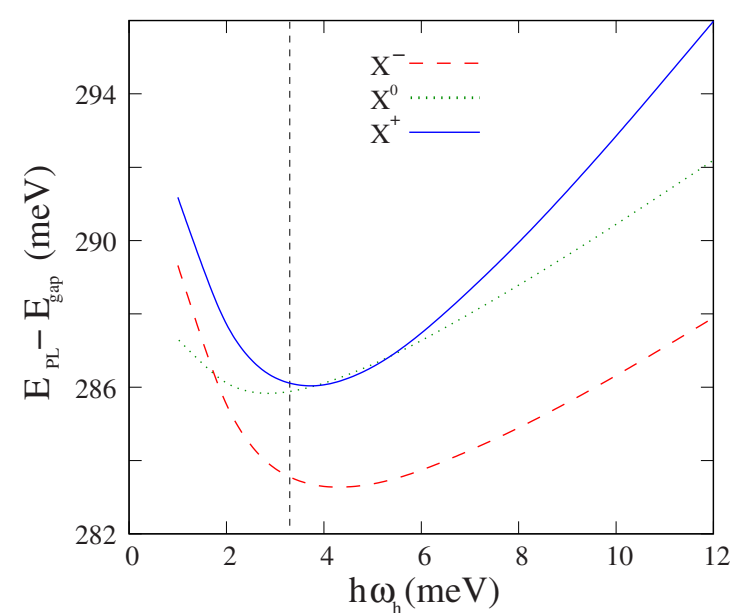

FIG. 5. (Color online) Recombination energies versus hole lateral confinement for a fixed electron confinement $\hbar \omega_{e}=30 \mathrm{meV}$. The dashed vertical line indicates the hole confinement leading to symmetric electron and hole wave functions. To the left (right) of the line, the hole is less (more) localized than the electron.

The qualitative differences in the recombination energies shown in Fig. 5 brought about by the electron-hole asymmetry, and ensuing changes in the regime of hole confinement, can be again rationalized in terms of the Coulomb contributions to Eq. (5). These are plotted in Fig. 6. For example, one can see that for very weak hole confinement $V_{e h}^{11} \sim V_{e e}>V_{e h}^{21}$, which explains the blueshift of $X^{-}\left[E_{\mathrm{PL}}\left(X^{-}\right)-E_{\mathrm{PL}}\left(X^{0}\right)=V_{e e}\right.$ $\left.-2 V_{e h}^{21}+V_{e h}^{11}>0\right]$. In addition, Fig. 6 gives a clear insight into the effect of hole correlations. While $V_{e e}$ is obviously insensitive to changes in the hole confinement, the other terms display a strong, nontrivial dependence. The dependence is especially strong for $V_{h h}$ and $V_{e h}^{12}$ because they involve two holes. As we move right from the symmetric electron-hole case (dashed vertical line), the hole confinement increases. At about $\hbar \omega_{h} \approx 6 \mathrm{meV} V_{h h}$ exceeds $V_{e e}$. This starts being consistent with a simple perturbational Coulomb picture ${ }^{42}$ and indicates that hole correlations are decreasing. Farther right, $V_{h h}$ overcomes all the electron-hole terms, meaning

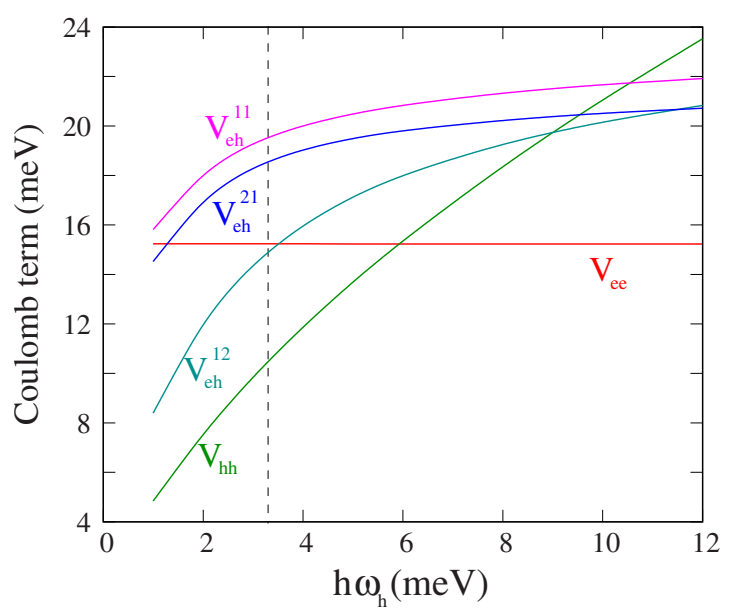

FIG. 6. (Color online) Absolute value of the Coulomb terms contributing to the exciton and trion states of Fig. 5. The dashed vertical line indicates the hole confinement where electron and hole wave functions are symmetric.

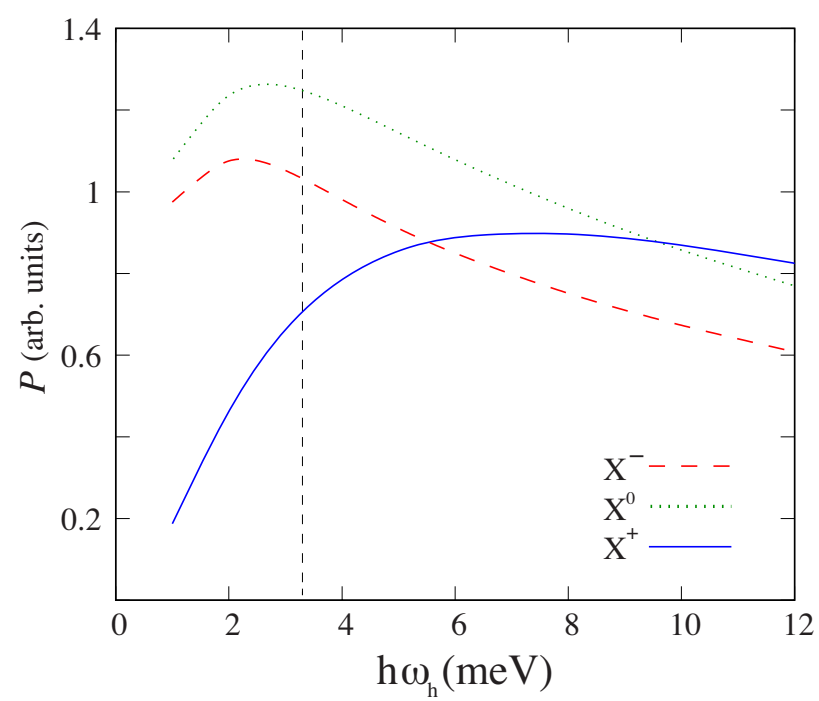

FIG. 7. (Color online) Recombination probability versus hole lateral confinement for the exciton and trion states of Fig. 3. The dashed vertical line indicates the hole confinement where electron and hole wave functions are symmetric.

that the hole configuration mixing is no longer able to maximize (minimize) the attraction (repulsion) terms enough to compensate for the single-particle electron-hole asymmetry, which renders $V_{e h}^{N_{N} N_{h}}$ smaller than $V_{h h}$.

Figure 7 shows the recombination probability of $X^{0}, X^{+}$, and $X^{-}$as a function of the hole confinement frequency. For $X^{0}$ and $X^{-}$the maximal PL is close to point of symmetric electron and hole wave functions (dashed vertical line), where the single-particle overlap is largest, and it decreases away from this point. Actually, the maximum is slightly shifted toward the left of the dashed line because $X^{0}$ and $X^{-}$ exhibit superradiant PL with increased Coulomb correlations (recall Fig. 3), which compensates for a moderate singleparticle asymmetry. The behavior is different for $X^{+}$because there is no superradiance and because the hole-hole correlation redistributes the charge density as shown in the left panel of Fig. 4, with an inner and outer hole surrounding the electron in the center. As the hole confinement strength increases, the two holes are squeezed toward the peak of the electron wave function. This increases the overlap and hence $\mathcal{P}\left(X^{+}\right)$. Note that this is contrary to the trend of $\mathcal{P}\left(X^{0}\right)$ and $\mathcal{P}\left(X^{-}\right)$. As a result, the sequence observed in Ref. 31 $\left[\mathcal{P}\left(X^{0}\right)>\mathcal{P}\left(X^{-}\right)>\mathcal{P}\left(X^{+}\right)\right]$holds only in the regime of weak and intermediate hole confinements $\left(\hbar \omega_{h}<6 \mathrm{meV}\right)$.

It follows from this section that the best fit of the experimental results of Ref. 31 is obtained when electrons and holes have similar lateral extension. For an electron with $\hbar \omega_{e}=30 \mathrm{meV}$, a symmetric hole wave function has $\hbar \omega_{h}$ $=3.33 \mathrm{meV}$. If we compare the corresponding two-electron and two-hole ground states, we find that the dominant configuration in both cases is the doubly-occupied $s$ shell. However, for electrons the weight of this configuration is $98.5 \%$ while for holes it is $53.5 \%$. The latter figure is even smaller than that of electrons in etched QDs with $\hbar \omega_{e} \approx 2 \mathrm{meV},{ }^{37,47}$ which gives an idea of the strength of hole correlations.

Finally we mention that we have run simulations assuming a lighter hole mass, $m_{h}^{*}=0.25$. This value, assuming iden- 


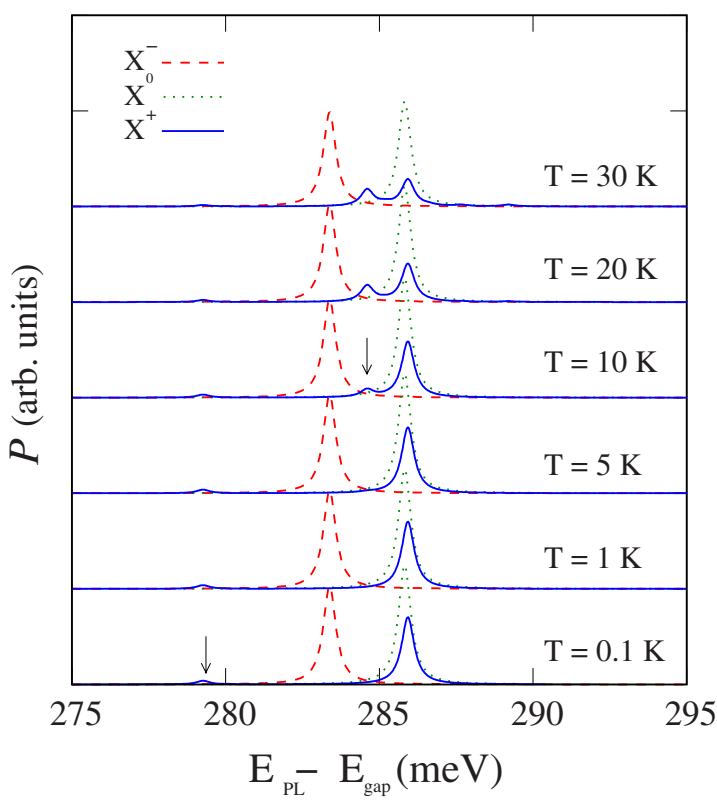

FIG. 8. (Color online) Emission spectrum of $X^{0}, X^{+}$, and $X^{-}$as a function of the temperature. The spectra are offset vertically. The arrow at $T=0.1 \mathrm{~K}$ points at the correlation-induced satellite of $X^{+}$ while the arrow at $T=10 \mathrm{~K}$ points at the resonance coming from the first-excited state of $X^{+}$, which has been activated thermally.

tical electron and hole wave function in the lateral direction, described well some experiments with InGaAs QDs. ${ }^{39}$ The same qualitative trends as found here were observed but the relative recombination energies did not match those measured in Ref. 31. Hole correlations were still important (for $\hbar \omega_{e}=30 \mathrm{meV}$, a symmetric hole required $\hbar \omega_{h}=6 \mathrm{meV}$, which gives a dominant two-hole configuration weight of $74 \%)$.

\section{Temperature dependence}

The markedly different energy structure of $X^{+}$as compared to $X^{0}$ and $X^{-}$reflects itself in the calculated temperature dependence of the PL spectra of the three excitonic species, shown in Fig. 8. The plot corresponds to a QD with $\hbar \omega_{e}=30 \mathrm{meV}$ and $\hbar \omega_{h}=3.33 \mathrm{meV}$ (symmetric electron and hole wave functions). In this figure, we consider not only the lowest excitonic transition but also a few excited ones which may acquire appreciable population with increasing temperature. For the temperatures under study however these transitions play a minor role only.

At the lowest temperature, one can see that $X^{+}$alone exhibits a weak satellite peak (pointed at by an arrow) well below the fundamental transition. This is due to the strong configuration mixing of holes, which introduces a sizeable contribution from the $s d_{z}$ configuration to the otherwise pure $s^{2}$ configuration of the two-hole ground state. This mixing enables the recombination of an $s$-shell electron with a $d$-shell hole. Similar features have been predicted for the excited states of excitons and multiexcitons, owing to electron-hole correlations, ${ }^{9,11}$ as well as for highly charged excitons. ${ }^{17}$ Here we show that for positive trions, such features show up for the ground state as well, this being a sig- nature of the stronger correlation regime. To our knowledge this resonance has not been explicitly reported in experiments but recent high-resolution PL measurements of single InGaAs QDs at low temperature $(T=5 \mathrm{~K})$ revealed a number of small satellite peaks below the fundamental transition of $X^{+}$which were however absent for $X^{0}$ and $X^{-}{ }^{-17}$

As the temperature increases, the population of the exciton or trion excited states increases at the expense of the ground state. For $X^{0}$ and $X^{-}$this has no observable results up to a few tens of Kelvin because the lowest-lying excitations are quite far in energy from the ground state (a few meV). However, for $X^{+}$-due to the larger density of statesmoderate temperatures suffice to yield visible changes in the spectrum. This can be seen in Fig. 8. With increasing temperature, the PL of the fundamental and the satellite peaks of $X^{+}$decreases, and a new resonance shows up at $\sim 284 \mathrm{meV}$ (indicated by an arrow at $T=10 \mathrm{~K}$ ). This resonance corresponds to the recombination of an $s$-shell electron and an $s$-shell hole, with a second hole remaining in the $p$ shell. The different sensitivity to temperature can be used as a means for distinguishing positively charged excitons from neutral and negative species. Temperature-dependent PL measurements in individual self-assembled InAs QDs seem consistent with this prediction, as they show a fast decrease in the fundamental $X^{+}$resonance and the appearance of a satellite peak right below it with increasing temperature [see Fig. 2(b) in Ref. 45].

\section{CONCLUSION}

Both our accurate effective-mass-FCI calculations and recent experimental observations of recombination energies and rates point to an important role of configuration mixing of valence-band holes in the dynamics of trions. Our results are in quantitative agreement with experimental finding if electrons and holes are taken to have similar lateral extension. Under such conditions, hole correlations are clearly non-negligible. Our results also show that in self-assembled (and weaker confined) QDs Coulomb correlations lead to $\left|V_{e h}^{N_{e} N_{h}}\right|>\left|V_{e e}\right|>\left|V_{h h}\right|$. More generally, the large increase in recombination probability of $X^{0}$ as a function of the dot size-a well-known result arising from the enhanced electron-hole Coulomb correlations, ${ }^{25-27}$-is suppressed almost completely for $X^{-}$and reversed for $X^{+}$, whose intensity decreases with dot size. This is due to the electron-electron and hole-hole Coulomb terms, which compensate (and overcome) the excitonic attraction. We also predict that signatures of the distinct hole energy structure can be found in the specific temperature dependence of the $X^{+}$spectrum.

\section{ACKNOWLEDGMENTS}

We are grateful to P. Dalgarno, F. Troiani, and M. Rontani for helpful discussions. We acknowledge financial support from the Italian Minister for Research through Contracts No. FIRB-RBIN06JB4C and No. PRIN-2006022932, from Cineca Calcolo Parallelo 2007-2008, from the Marie Curie Project Contract No. MEIF-CT-2006-023797, and from the Ramon y Cajal program. 
*climente@unimo.it

${ }^{1}$ Z. I. Alferov et al., Nano-Optoelectronics: Concepts, Physics and Devices, edited by M. Grundmann (Springer-Verlag, Berlin, 2002).

${ }^{2}$ For a review, see, e.g., V. Zwiller, T. Aichele, and O. Benson, New J. Phys. 6, 96 (2004).

${ }^{3}$ H. J. Krenner, S. Stufler, M. Sabathil, E. C. Clark, P. Ester, M. Bichler, G. Abstreiter, J. J. Finley, and A. Zrenner, New J. Phys. 7, 184 (2005).

${ }^{4}$ G. Chen, N. H. Bonadeo, D. G. Steel, D. Gammon, D. S. Katzer, D. Park, and L. J. Sham, Science 289, 1906 (2000).

${ }^{5}$ E. Biolatti, R. C. Iotti, P. Zanardi, and F. Rossi, Phys. Rev. Lett. 85, 5647 (2000).

${ }^{6}$ F. Troiani, U. Hohenester, and E. Molinari, Phys. Rev. B 62, R2263 (2000); F. Troiani, E. Molinari, and U. Hohenester, Phys. Rev. Lett. 90, 206802 (2003).

${ }^{7}$ A. Kiraz, M. Atatüre, and A. Immamoglu, Phys. Rev. A 69, 032305 (2004).

${ }^{8}$ E. A. Stinaff, M. Scheibner, A. S. Bracker, I. V. Ponomarev, V. L. Korenev, M. E. Ware, M. F. Doty, T. L. Reinecke, and D. Gammon, Science 311, 636 (2006).

${ }^{9}$ L. Jacak, P. Hawrylak, and A. Wojs, Quantum Dots (SpringerVerlag, Berlin, 1998).

${ }^{10} \mathrm{Ph}$. Lelong and G. Bastard, Solid State Commun. 98, 819 (1996).

${ }^{11}$ U. Hohenester and E. Molinari, Phys. Status Solidi A 178, 277 (2000); Phys. Status Solidi B 221, 19 (2000).

${ }^{12}$ A. J. Williamson, A. Franceschetti, and A. Zunger, Europhys. Lett. 53, 59 (2001).

${ }^{13}$ J. Shumway, A. Franceschetti, and A. Zunger, Phys. Rev. B 63, 155316 (2001).

${ }^{14}$ G. Bester and A. Zunger, Phys. Rev. B 68, 073309 (2003).

${ }^{15}$ Y. Turki-Ben Ali and R. Bennaceur, Physica E (Amsterdam) 28, 347 (2005)

${ }^{16}$ A. Hartmann, Y. Ducommun, E. Kapon, U. Hohenester, and E. Molinari, Phys. Rev. Lett. 84, 5648 (2000).

${ }^{17}$ M. Ediger, G. Bester, A. Badolato, P. M. Petroff, K. Karrai, A. Zunger, and R. J. Warburton, Nat. Phys. 3, 774 (2007).

${ }^{18}$ D. V. Regelman, E. Dekel, D. Gershoni, E. Ehrenfreund, A. J. Williamson, J. Shumway, A. Zunger, W. V. Schoenfeld, and P. M. Petroff, Phys. Rev. B 64, 165301 (2001).

${ }^{19}$ J. J. Finley, M. Sabathil, P. Vogl, G. Abstreiter, R. Oulton, A. I. Tartakovskii, D. J. Mowbray, M. S. Skolnick, S. L. Liew, A. G. Cullis, and M. Hopkinson, Phys. Rev. B 70, 201308(R) (2004).

${ }^{20}$ G. A. Narvaez, G. Bester, and A. Zunger, Phys. Rev. B 72, 245318 (2005).

${ }^{21}$ L. Landin, M. S. Miller, M. E. Pistol, C. E. Pryor, and L. Samuelson, Science 280, 262 (1998); L. Landin, M. E. Pistol, C. Pryor, M. Persson, L. Samuelson, and M. Miller, Phys. Rev. B 60, 16640 (1999).

${ }^{22}$ M. Lomascolo, A. Vergine, T. K. Johal, R. Rinaldi, A. Passaseo, R. Cingolani, S. Patanè, M. Labardi, M. Allegrini, F. Troiani, and E. Molinari, Phys. Rev. B 66, 041302(R) (2002).

${ }^{23}$ S. Rodt, A. Schliwa, K. Pötschke, F. Guffarth, and D. Bimberg, Phys. Rev. B 71, 155325 (2005).

${ }^{24}$ R. Rinaldi, S. Antonaci, M. DeVittorio, R. Cingolani, U. Hohenester, E. Molinari, H. Lipsanen, and J. Tulkki, Phys. Rev. B
62, 1592 (2000).

${ }^{25}$ G. W. Bryant, Phys. Rev. B 37, 8763 (1988).

${ }^{26}$ W. Que, Phys. Rev. B 45, 11036 (1992).

${ }^{27}$ S. Corni, M. Brasken, M. Lindberg, J. Olsen, and D. Sundholm, Phys. Rev. B 67, 045313 (2003).

${ }^{28}$ M. Wimmer, S. V. Nair, and J. Shumway, Phys. Rev. B 73, 165305 (2006).

${ }^{29}$ G. Bacher, R. Weigand, J. Seufert, V. D. Kulakovskii, N. A. Gippius, A. Forchel, K. Leonardi, and D. Hommel, Phys. Rev. Lett. 83, 4417 (1999).

${ }^{30}$ C. Santori, G. S. Solomon, M. Pelton, and Y. Yamamoto, Phys. Rev. B 65, 073310 (2002).

${ }^{31}$ P. A. Dalgarno, J. M. Smith, J. McFarlane, B. D. Gerardot, K. Karrai, A. Badolato, P. M. Petroff, and R. J. Warburton, Phys. Rev. B 77, 245311 (2008).

${ }^{32}$ S. J. Cheng, Phys. Rev. B 76, 075329 (2007).

${ }^{33}$ M. Atatüre, J. Dreiser, A. Badolato, A. Högele, K. Karrai, and A. Imamoglu, Science 312, 551 (2006).

${ }^{34}$ B. D. Gerardot, D. Brunner, P. A. Dalgarno, P. Öhberg, S. Seidl, M. Kroner, K. Karrai, N. G. Stoltz, P. M. Petroff, and R. J. Warburton, Nature (London) 451, 441 (2008).

${ }^{35}$ A. Ebbens, D. N. Krizhanovskii, A. I. Tartakovskii, F. Pulizzi, T. Wright, A. V. Savelyev, M. S. Skolnick, and M. Hopkinson, Phys. Rev. B 72, 073307 (2005).

${ }^{36}$ M. Bayer, G. Ortner, O. Stern, A. Kuther, A. A. Gorbunov, A. Forchel, P. Hawrylak, S. Fafard, K. Hinzer, T. L. Reinecke, S. N. Walck, J. P. Reithmaier, F. Klopf, and F. Schäfer, Phys. Rev. B 65, 195315 (2002).

${ }^{37}$ S. Kalliakos, C. P. Garcia, V. Pellegrini, M. Zamfirescu, L. Cavigli, M. Gurioli, A. Vinattieri, A. Pinczuk, B. S. Dennis, L. N. Pfeiffer, and K. W. West, Appl. Phys. Lett. 90, 181902 (2007).

${ }^{38}$ A. Wojs, P. Hawrylak, S. Fafard, and L. Jacak, Phys. Rev. B 54, 5604 (1996).

${ }^{39}$ S. Raymond, S. Studenikin, A. Sachrajda, Z. Wasilewski, S. J. Cheng, W. Sheng, P. Hawrylak, A. Babinski, M. Potemski, G. Ortner, and M. Bayer, Phys. Rev. Lett. 92, 187402 (2004).

${ }^{40}$ M. Rontani, C. Cavazzoni, D. Bellucci, and G. Goldoni, J. Chem. Phys. 124, 124102 (2006).

${ }^{41}$ B. Szafran, B. Stébé, J. Adamowski, and S. Bednarek, Phys. Rev. B 66, 165331 (2002).

${ }^{42}$ R. J. Warburton, B. T. Miller, C. S. Dürr, C. Bödefeld, K. Karrai, J. P. Kotthaus, G. Medeiros-Ribeiro, P. M. Petroff, and S. Huant, Phys. Rev. B 58, 16221 (1998).

${ }^{43}$ Although the experiment reports recombination rates rather than probabilities, the former are directly proportional to the latter (see, e.g., Ref. 28) so that the relative values are the same.

${ }^{44}$ E. Hanamura, Phys. Rev. B 37, 1273 (1988); 38, 1228 (1988).

${ }^{45}$ N. I. Cade, H. Gotoh, H. Kamada, H. Nakano, and H. Okamoto, Phys. Rev. B 73, 115322 (2006).

${ }^{46}$ B. Szafran, T. Chwiej, F. M. Peeters, S. Bednarek, and J. Adamowski, Phys. Rev. B 71, 235305 (2005).

${ }^{47}$ C. P. Garcia, V. Pellegrini, A. Pinczuk, M. Rontani, G. Goldoni, E. Molinari, B. S. Dennis, L. N. Pfeiffer, and K. W. West, Phys. Rev. Lett. 95, 266806 (2005); S. Kalliakos, M. Rontani, V. Pellegrini, C. P. Garcia, A. Pinczuk, G. Goldoni, E. Molinari, L. N. Pfeiffer, and K. W. West, Nat. Phys. 4, 467 (2008). 